\title{
Structure and luminescence study of nanoporous silicon layers with high internal surface
}

\author{
V.A. Makara ${ }^{1}$, M.M. Melnichenko ${ }^{1}$, K.V. Svezhentsova ${ }^{1}$, L.Yu. Khomenkova ${ }^{2}$, O.M. Shmyryeva ${ }^{3}$ \\ ${ }^{\prime}$ Taras Shevchenko Kiev National University, 6, prospect Akad. Glushkova, 03127 Kyiv, Ukraine, realcrystallab@univ.kiev.ua \\ ${ }^{2} V$. Lashkaryov Institute of Semiconductor Physics NAS of Ukraine, 41, prospect Nauky, 03028 Kyiv, Ukraine \\ ${ }^{3}$ National Technical University of Ukraine "KPI", 37, prospect Peremogy, 03056 Kiev, Ukraine
}

\begin{abstract}
In this work, the technique of formation of homogeneous nanoporous silicon layers with high internal surface on solar cell substrates by stain etching is developed. Emission and structure properties of such layers were studied by photoluminescence (PL), photoluminescence excitation, Auger electronic spectroscopy, atomic force microscopy and scanning tunnel microscopy methods. It was observed that nanoporous layers are of high homogeneity and their thickness is $\sim 20-25 \mathrm{~nm}$. It was shown that the higher PL intensity of such layers in comparison with PL intensity of the layers prepared on standard substrates in the same regimes is connected with the higher internal surface. The increase of PL intensity during prolonged aging in air at the room temperature was observed.
\end{abstract}

Keywords: porous silicon, stain etching, nanoporous layer.

Paper received 26.09.03; accepted for publication 11.12.03.

\section{Introduction}

One of the unique properties of porous silicon (PS) is high ratio of its internal surface to its volume. It is known that this material is sensitive to different environment, which allows to produce various type sensors based on porous silicon [1,2]. At the same time, to detect some molecule reactions, it is neccessary to use macroporous layers due to micrometer scale of molecules while nanoporous layers can be used for gas sensors. It should be noted that these layers will be homogeneous in all the porous area.

Different techniques were proposed for creation of porous layers. The most widespread are electrochemical etching in HF-based solutions and stain etching in $\mathrm{HF}: \mathrm{HNO}_{3}$ solution. The variation of electrochemical conditions (current density, time, composition of electrolyte) allow to change silicon crystallite sizes in the wide range and vary the thickness of layers from $100 \mathrm{~nm}$ to $100 \mu \mathrm{m}$ [3]. But this method has some essential drawbacks. One of them is using the back contact that allows to form porous layers only on one side of substrates and makes difficulties in the treatments of silicon substrates with large qreas. Besides, in this case, homogeneity of porous layers can be disturbed at the edges of layers.

The stain etching technique allows to avoid these drawbacks and form porous layers on both sides of high square substrates. This method is more simple, and it does not demand special devices. However, it is impossible to made thick layers due to competition between pore formation and polishing processes, which results in lower internal surface area in comparison with layers formed by electrochemical etching.

So, this work is devoted to the development of a technique to form homogeneous porous layers with large internal surface by stain etching as well as to study layer properties.

\section{Experimental procedure}

Two types of porous layers were formed on monocrystal silicon substrates of $p$-type, B-doped, (100) orientation, resistivity of $1 \Omega \cdot \mathrm{cm}$ and thickness of about $300 \mu \mathrm{m}$. Set-A layers were produced on the substrates with mirrored surface on one or both sides after mechanical and chemical polishing. Set B samples were made on the wafers for solar cells. Such substrates were not polished after standard procedure of chemical cleaning for solar cells and usually have dull surface. Before PS layers formation, all substrates were processed in water $50 \% \mathrm{KOH}$ solution wafers to remove of superficial pollution and previous loosening of surfaces. The square of the substrates was $100 \mathrm{~cm}^{2}$. 


\section{V.A. Makara et al.: Structure and luminescence study of nanoporous silicon layers...}

PS layers were prepared by stain etching in $\mathrm{HF}: \mathrm{HNO}_{3}$ solution at the room temperature, natural day-time illumination and time duration from 1 to $5 \mathrm{~min}$ [4]. The layers were formed on both sides of the substrates.

The structure of PS surface was studied using atomic force microscope (AFM) Nanoscope IIIa in "tapping mode" and scanning tunnel microscope (STM).

The Auger electronic spectroscopy (AES) measurements were carried out by LAS Riber-2000 system. Energy of a primary electron beam was $3 \mathrm{KeV}$, the current of a probe was $5 \times 10^{-7} \mathrm{~A}$. Argon ion etching was used to control depth distribution of elements in PS layers. The energy of ions was $4 \mathrm{keV}$. Etching rate was $3 \mathrm{~nm}$ per minute. The information was obtained from a $100 \times 100 \mu \mathrm{m}^{2}$ surface.

Photoluminescence (PL) was excited by a xenon-150 lamp with a grating monochromator MDR-23, dispersed by a prism spectrometer IKS-12 and detected by a photomultiplier FEU-79. All spectra were corrected on spectral response. The PL excitation (PLE) spectra were measured at the maximum of PL band. All measurements were performed at $300 \mathrm{~K}$.

\section{Results and discussion}

The AFM investigations of substrates for set-B samples shown that the surface of such substrates has the roughness $\sim 1 \mu \mathrm{m}$ (Fig. 1,a), while the roughness of substrates for set-A layers was $\sim 50 \mathrm{~nm}$ (with mirrored surface). Morphology of surface PS layers on set-A samples was in detail investigated in many papers [5-7].

Porous layers produced on both types of substrates had the ordered structure and repeated the surface morphology of initial substrates both for set-A samples and for the set-B ones. A representative surface morphology of PS layers on set-B substrates is depicted in Fig.1,b. The analysis of obtained AFM images shows that PS layers contain the hillocks with height $\sim 20 \mathrm{~nm}$. These hill- ocks apparently correspond to the vertices of Si crystallites, whereas the hollows between them correspond to the outcrop of narrow (nanometer) pores on the surface. A similar structure is also observed on all the relief of the surface substrate and strictly repeats morphology of the surface of monocrystalline silicon, i.e., the microrelief of silicon substrates is modulated with the nanorelief.

It is known that because of the certain size of AFM tip the information on the depth of PS layer could be incorrect in the case of deep narrow pores. Such pores can be displayed as shallow holes. So, STM investigation of PS layer structures on B-set samples were performed. This method allows to study separate pores of porous silicon.

The STM images obtained on PS area of $110 \times 110 \mathrm{~nm}^{2}$ and $20 \times 20 \mathrm{~nm}^{2}$ are shown on Fig. 2 . As one can see, the hillocks are clearly resolved and their average diameters are of a few nanometers. The study of height profile shows that the height of hillocks is $\sim 20 \mathrm{~nm}$, which well correlates with AFM data.

It should be noted that structure investigation of different parts of porous layers prepared on high square substrates showed that these layers were stably reproduced in morphology of these PS surfaces. It is necessary to note that between the samples separately prepared under identical conditions it was not observed any appreciable difference in the structure of their surfaces.

Additional information about PS thickness was obtained from AES investigation of the layers. Fig. 3, $a$ shows typical AES depth profile of the PS samples. Curves $1,2,3$ and 4 in the figure correspond to the concentration distributions of $\mathrm{Si}, \mathrm{O}, \mathrm{C}$ and $\mathrm{SiO}_{\mathrm{x}}$ with the $\mathrm{Ar}^{+}$sputtering time, respectively. Absence of the contents $\mathrm{N}$ and $\mathrm{F}$ that frequently meet in AES spectra of stain etching PS, shows good washing and drying the samples. Comparing the curves 1, 2, 3 and 4, the content of $\mathrm{Si}, \mathrm{O}, \mathrm{C}$ and $\mathrm{SiO}_{\mathrm{x}}$ in $\mathrm{PS}$ sample surface is approximately $0.5,24.1,49.6$ and $25.8 \%$, respectively. As one can see, $\mathrm{SiO}_{\mathrm{x}}$ content de-
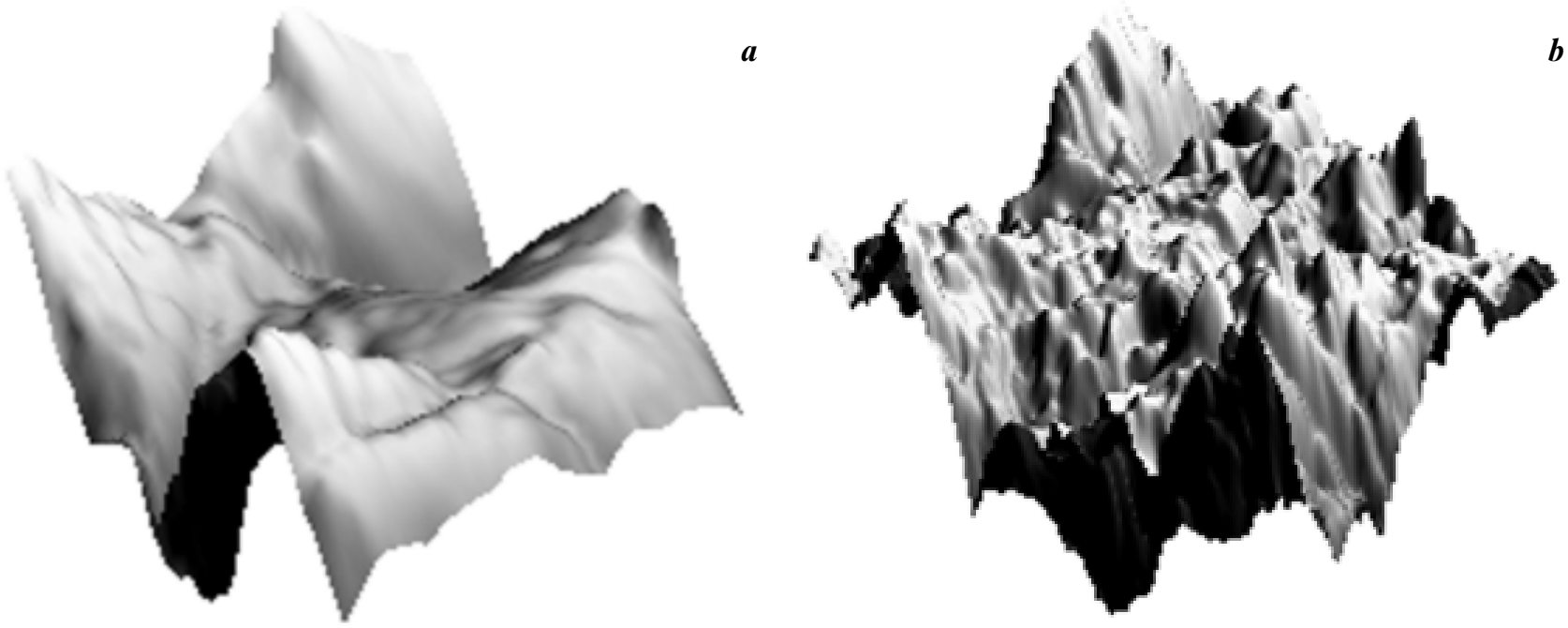

Fig. 1. AFM images of monocrystalline silicon substrate (a) and PS surface for set-B samples (b), (the area of scanning $1 \times 1 \mu \mathrm{m}{ }^{2}$ ). 


\section{V.A. Makara et al.: Structure and luminescence study of nanoporous silicon layers...}
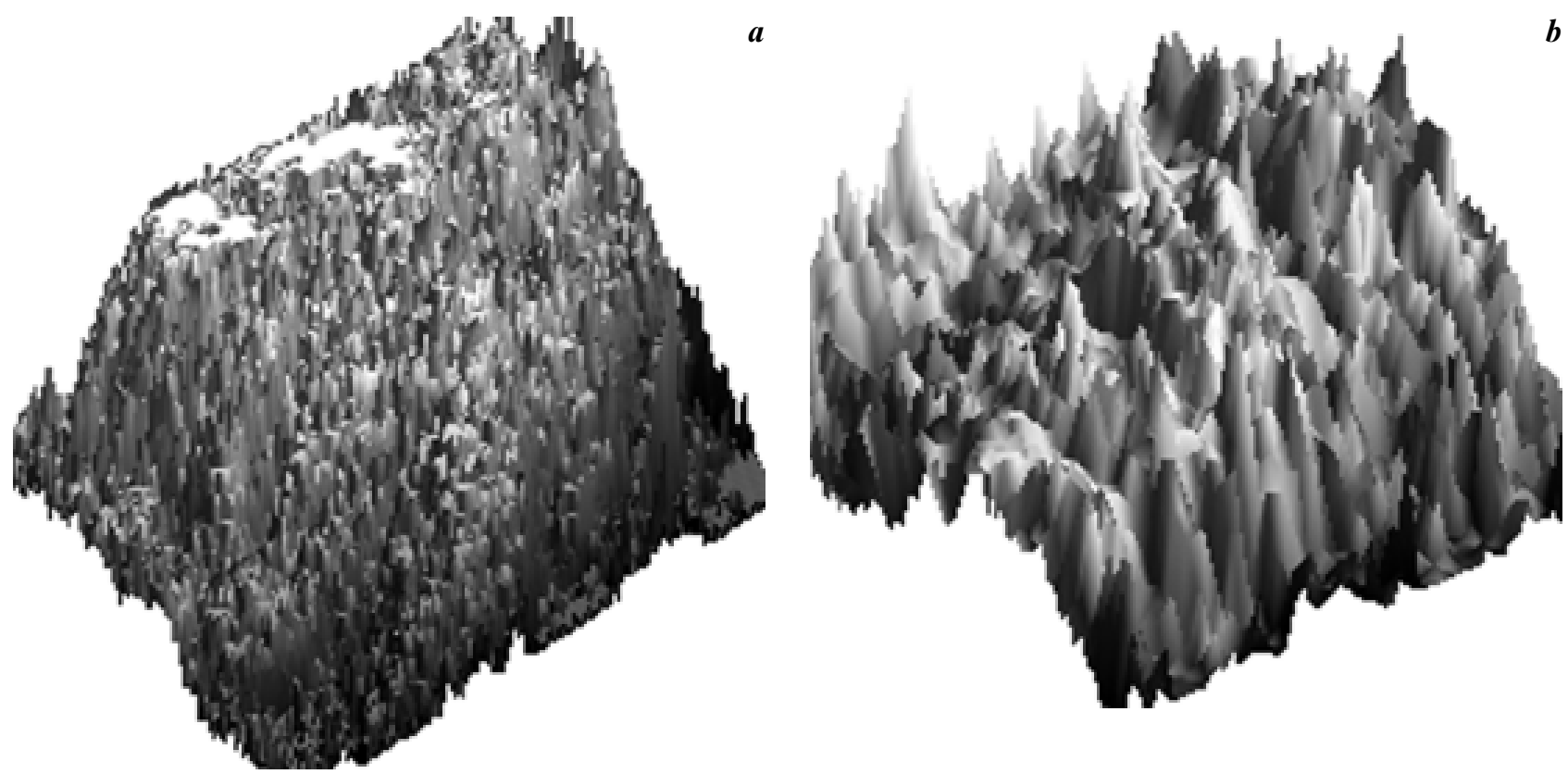

Fig. 2. STM images of the porous silicon surface, the scanned area is $110 \times 110 \mathrm{~nm}^{2}$ (a), $20 \times 20 \mathrm{~nm}^{2}$ (b).

creases steadily up to its complete disappearance after argon ion etching for $7 \mathrm{~min}$, which corresponds to $\sim 20$ $25 \mathrm{~nm}$ depth. At the same time, the content of Si increases up to its maximum value at the same depth. So, we can conclude that the thickness of nanoporous layer is approximately $\sim 20-25 \mathrm{~nm}$. Since content of $\mathrm{O}$ and $\mathrm{C}$ does not disappear entirely, we can suppose that some deep pores can be present in the layers and total thickness of porous layer is more than 20-25 nm. For comparison, we submitted the typical AES depth profiles of the silicon substrates (Fig. 3,b).

The typical PL spectra of set-A and set-B samples are shown in Fig. 3. As one can see, PL is excited by the light from $280-530 \mathrm{~nm}$ spectral region (Fig. 3, curves 1, 2). It should be noted that different parts of PS samples prepared at high square substrates have the similar PL spectra (curves 3 to 5 for the set-A samples and curves 6 to 8 for the set-B samples). Their intensities and peak positions differ insignificantly. Besides, the samples with small squares prepared separately at the same etching regime showed the similar luminescent properties, too. So, we can compare different samples prepared in the same etching regime and testify about high reproducibility of the method. It should be noted that it is completely correct, since all spectra were measured on one setup under identical conditions.

The comparison of PL properties of the set-A and setB samples shows that PL and PLE spectra of the set-B
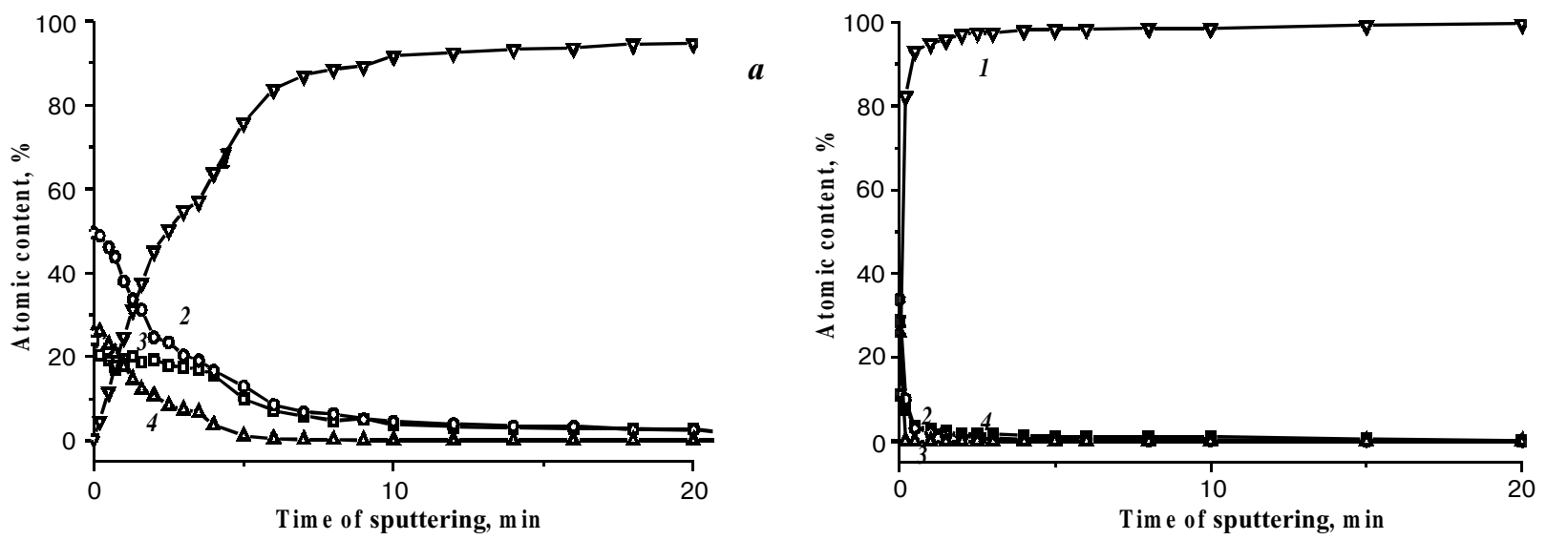

Fig. 3. AES depth profile of PS sample (a) and silicon substrates (b). 1,2,3, and 4 stand for concentrations distribution of Si, O, C and $\mathrm{SiO}_{\mathrm{x}}$, respectively. 


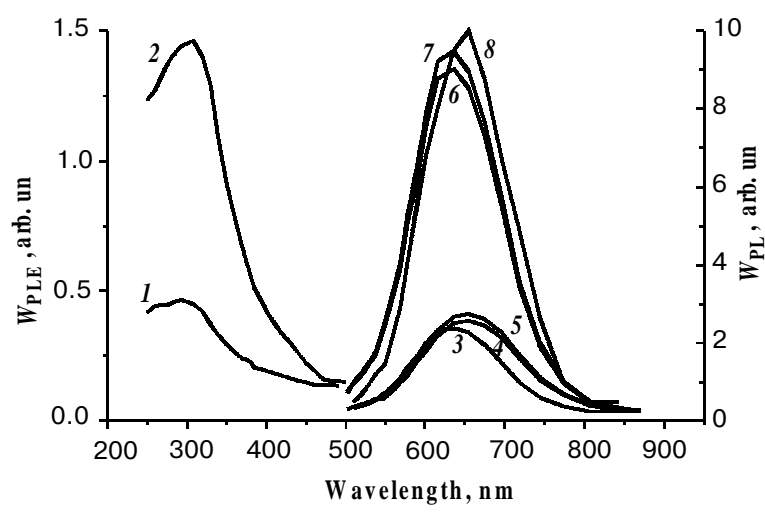

Fig. 4. PLE $(1,2)$ and PL (3-8) spectra of PS layers received by stain etching on set-A $(1,3-5)$ and set-B $(2,6-8)$ samples. Excitation wavelength in PL measuruments was $320 \mathrm{~nm}$.

samples (porous layers prepared on substrates for solar cells) are similar to set-A ones. At the same time in the contrast of the set-A layers PL intensity of the set-B samples is higher than set-A samples emission and is enough for supervision PL to the naked eye. This PL intensity is close to the one of the PS layers prepared by electrochemical etching.

The reason of higher PL intensity of set-B samples in comparison with the set-A ones is as follows. Since the sizes of hillocks are similar for both type PS layers, we can think that the higher PL intensity of PS layers prepared on set-B substrates is connected with their high porous area. As one can see from AFM investigation, the surface of initial set-B substrate was more developed that of the set A at the same volume. So, the area of PS layer formed on set-B substrates will be larger than area of the layer prepared on set-A wafers.

The main lack for application of PS in optoelectronics is instability of its luminescent properties and degradation of luminescence during aging in air. Since the moment of PS visible luminescence discovery, many works have been done to research PL aging.

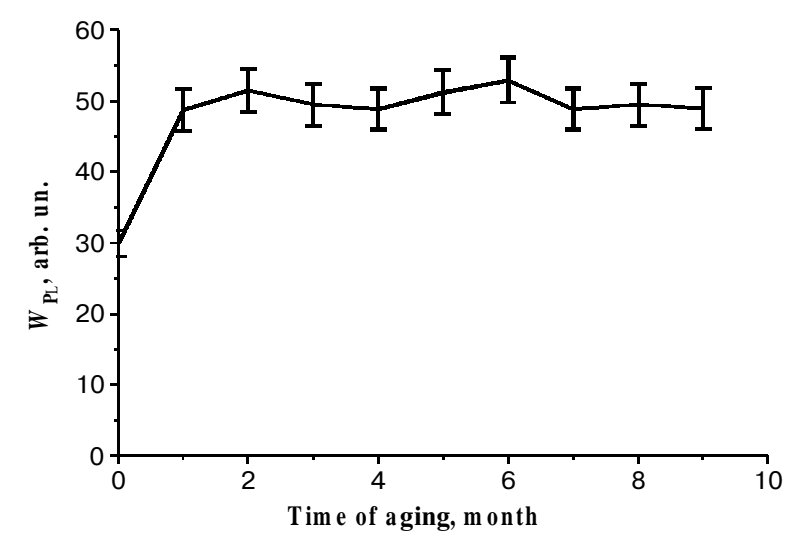

Fig. 5. Dependence of intensity PL on time of aging of PS thin layers in air, excitation wavelength in PL measuruments was $337 \mathrm{~nm}$.
As for our case, PL intensity of both type samples increased with time (Fig. 5). The changes in PL intensity were estimated via changes of its intensity at the maximum. During one-month aging, PL intensity increased by 1.5 to 2 times due to oxidation process, which is in agreement with the data obtained by different authors $[8,9]$. After one-month aging, PL intensity reached its maximum and then remained constant for 10 months. Some deviation of PL intensity from the observed constant value within $5-7 \%$ limits is probably caused by different positions of points for measurements on the sample surface. It should be noted that PL peak position did not change practically during aging.

In conclusion, PS layers with high internal surface prepared by stain etching on monocrystalline silicon substrates for solar cells were studied. It was shown that these layers are of high homogeneity and stably reproduced from the point view of their surface morphology. It was found that the thickness of nanoporous layers were $\sim 20-25 \mathrm{~nm}$. These layers prepared on solar cells substrates have bright PL. Its intensity is higher that PL intensity of PS layers formed on the standard substrates at the same regimes and is enough to observe with a naked eye. It should be noted that PL intenisty of PS layers increased significantly during one-month aging in air at the room temperature and then is stable for 2 to 10 months. It should be noted that nanoporous thin (20-25 nm) layers with the high porous area can be used for application in various very sensitive devices, such as gas detectors, solar cells, biosensors and others.

\section{References}

1. A.G. Cullis, L.T. Canc ham, P.D. Calcott, The structural and luminescence properties of porous silicon // Applied Physics Reviews, 82, P. 909-965 (1997).

2. M.Y. Ghannam, A.A. Abouelsaood, J.F. Nijs, A semiquantitative model of a porous silicon layer used as a light diffuser in a thin film solar cell. // Solar Energy Materials \&Solar Cells, 60, P. 105-125 (2000).

3. Y. Arita, Y. Sunohara, Formation and prorerties of porous silicon films // J. Electrochem. Soc. 124 (2), P.285-294 (1977).

4. S.Kalem, M. Rosenbauer, Optical and structural investigation of stain-etched silicon // Appl. Phis. Lett., 67(17), P. 25511553 (1995).

5. S. Shin, K.H. Jung, T.Y. Hsieh, J. Sarathy, J.C. Campbell, D.L. Kwong, Photoluminescence and formation mechanism of chemically etched silicon // Appl. Phis. Lett., 60(15), P. 1863-1865 (1992).

6. V.M. Starodub, L.L. Fedorenko, G.V. Beketov, N.F. Starodub, Obtaining, characterization and use of porous silicon for biosensing purposes // Abstr. of Conf. Porous Semiconductors - Science and Technologi, P. 108-110 (1998)

7. N.E. Korsunskaya, E.B. Kaganovich, L.Yu. Khomenkova, B.M. Bulach, B.R. Dzumaev, G.V. Beketov, E.G. Manoilov, Effect of adsorbtion and desorption on photoluminescence excitation spectra of porous silicon // Applied Surface Science, 166, P.349-353 (2000).

8. Y.M. Weng, Z.N. Fan, X.F. Zong, Luminescence studies of porous silicon. // Appl. Phis. Lett., 63(2), P.168-170 (1993).

9. P.D.Stevens, R. Glosser, Anomalous photoluminescense behavior of porous silicon // Appl. Phis. Lett., 63(6), P.803805 (1993). 